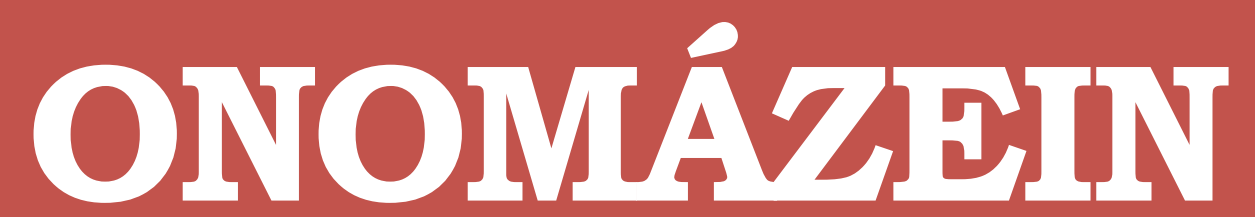

Revista de lingüística, filología y traducción
PONTIFICIA UNIVERSIDAD

\title{
La escritura de crónicas periodísticas informativas: una propuesta de avance desde el feedback correctivo escrito
}

The writing of informative journalistic chronicles: an advance proposal from written corrective feedback

\section{Steffanie Kloss Medina ${ }^{1}$}

Universidad Católica de la Santísima Concepción

Chile

\section{Anita Ferreira Cabrera ${ }^{2}$ \\ Universidad de Concepción \\ Chile}

\section{(c) $(i)$}

Steffanie Kloss Medina: Universidad Católica de la Santísima Concepción, Chile. | E-mail: skloss@ucsc.cl Anita Ferreira Cabrera: Universidad de Concepción, Chile. | E-mail: aferreir@udec.cl 


\section{Resumen}

El objetivo de este estudio es medir el efecto del feedback correctivo escrito (FCE) directo y metalingüístico en el uso adecuado de marcadores discursivos con valor organizativo en crónicas periodísticas informativas para mejorar la precisión lingüística. Dichos marcadores son relevantes para la organización y conexión de crónicas informativas, las que poseen una textura en que predomina la narración con una modalidad cronológica. Para ello se llevó a cabo un estudio experimental con pretest, post-test inmediato y post-test diferido. La muestra corresponde a 39 sujetos, divididos en dos grupos experimentales y un grupo control. Los grupos experimentales (GE) recibieron sistemáticamente las estrategias de FCE directo (GE1) y FCE metalingüístico (GE2), mientras que el grupo control (GC) no recibió feedback. Los resultados evidencian los efectos positivos de las estrategias de feedback correctivo seleccionadas para incrementar el aprendizaje y uso correcto de marcadores discursivos con valor organizativo en crónicas periodísticas.

Palabras clave: feedback correctivo escrito metalingüístico y directo; marcadores discursivos con valor organizativo; crónica periodística.

\section{Abstract}

The objective of this study is to measure the effect of direct and metalinguistic written corrective feedback (WCF) on the proper use of discursive markers with organizational value in informative journalistic chronicles in order to improve linguistic accuracy. These markers are relevant for the organization and connection of informative chronicles that have a texture in which narration predominates with a chronological modality. To this aim, an experimental study was carried out with pretest, immediate post-test and delayed post-test. The sample corresponds to 39 subjects, divided into two experimental groups and a control group. The experimental groups (EG) systematically received the strategies of direct WCF (EGI) and metalinguistic WCF (EG2), while the control group (CG) did not receive feedback. The results show the positive effects of the corrective feedback strategies selected to increase the learning and the correct use of discursive markers with organizational value in journalistic chronicles.

Keywords: metalinguistic and direct written corrective feedback; discursive markers with organizational value; journalistic chronicle.

1 La autora agradece el financiamiento de CONICYT-CHA/Doctorado Nacional-2014- 21140533.

2 El estudio que se presenta en este artículo se ha desarrollado en el contexto del proyecto de investigación FONDECYT- CONICYT No. 1140651 "El feedback correctivo escrito directo e indirecto en la adquisición y aprendizaje del español como lengua extranjera". 


\section{Introducción}

Las habilidades de lectura y escritura son dos variables relevantes para la educación superior. En ese sentido, las universidades chilenas, mediante programas de acompañamiento, han tratado de fomentar y formar habilidades en escritura para estudiantes novatos.

Hace más de una década, Paula Carlino inició un proceso de estudio sobre alfabetización académica que se cristalizó con "Escribir y leer en la universidad: responsabilidad compartida entre alumnos, docentes e instituciones" (2004), en el cual describió la relevancia de la alfabetización académica para normar el proceso de inicio en la educación universitaria. Posteriormente, Carlino (2013) realiza una investigación denominada "Alfabetización académica 10 años después", en la que se pregunta qué ha ocurrido una vez que ha sido diagnosticado el problema de la falta de alfabetización disciplinar.

Los resultados de Carlino $(2004,2013)$ están en concordancia con lo que ocurre en Chile, pues si bien es cierto que se ha tratado de apoyar los procesos de inserción de estudiantes novatos en los ámbitos de mayor complejidad académica, mediante diversos programas organizados en las universidades (CIAE en la Universidad de Chile, CADE en la Universidad de Concepción, CEADE en la Universidad Católica de la Santísima Concepción, CIADE en la Universidad Andrés Bello, entre otros), estos aún no son extensivos a todas las universidades ni tampoco a todos los estudiantes, ya que son reducidos los grupos de profesionales especialistas en el ámbito académico disciplinar que manejen la metodología y que, además, sean conscientes del problema.

La Universidad de Concepción, mediante el Centro de Apoyo al Desarrollo del Estudiante (CADE), realiza talleres a alumnos de primer año, pero no cuenta con lo esencial, estrategias focalizadas en géneros discursivos de la especialidad, es decir, géneros de las ciencias, matemáticas, humanidades para apoyar los procesos de escritura.

En este contexto, el estudio que se presenta consiste en una intervención lingüística de 10 semanas, en la que estudiantes de primer año de Periodismo realizaron tareas de escritura una vez a la semana. Dichas tareas consistían en la redacción de crónicas periodísticas en la plataforma Moodle (ARCOUdeC); posteriormente, las crónicas eran corregidas con estrategias de feedback correctivo escrito (FCE) dispuestas para cada grupo (GE1, GE2) por la docente responsable del curso y por dos ayudantes de la asignatura, quienes revisaban todas las correcciones de manera conjunta.

La estrategia para corregir los textos correspondió al feedback correctivo escrito (FCE) en dos modalidades: directo y metalingüístico (Ellis, 2009). La finalidad de esta intervención es mejorar la precisión lingüística, mediante el uso adecuado de marcadores discursivos con valor organizativo (MDO en adelante). Es relevante abordar MDO, porque estos tienen la función de unir y articular el texto y, a la vez, beneficiar a la superestructura textual, mediante la textura narrativa cronológica que caracteriza a este género. 
El siguiente artículo se estructura de la siguiente manera: en primer lugar, mediante un marco teórico de referencia para presentar los conceptos fundamentales que nutren esta investigación; luego, en la dimensión metodológica se presenta el diseño de la investigación; posteriormente, se entrega el análisis y los resultados de la intervención lingüística; finalmente, se presentan las conclusiones, proyecciones y limitaciones.

\section{Fundamentos teóricos}

El marco teórico de referencia de este trabajo se presenta de la siguiente forma: en primer lugar, se presentará la corrección de la escritura y la relevancia del feedback correctivo escrito, según las distintas estrategias y la operacionalización de estas, y, en segundo lugar, se definirá la forma lingüística a tratar en este estudio (MDO).

\subsection{Feedback correctivo escrito (FCE)}

Se ha utilizado una terminología diferente para definir y poner en práctica el feedback correctivo. Para clarificar este concepto es propicio señalar que surge como evidencia negativa, a partir de la exposición a una lengua distinta a la materna, es decir, un estudiante que se expone al aprendizaje de una L2. Cabe mencionar que esa evidencia en el contexto de práctica puede ser positiva o negativa; sin embargo, esta evidencia positiva solo proporciona a los estudiantes modelos de lo que es posible y gramaticalmente aceptable, y la evidencia negativa entrega a los alumnos información sobre lo que es inaceptable en L2 (Long, 1996, 2007). En una segunda instancia, Chaudron (1977) utiliza el término "corrección de errores" para referirse a movimientos correctivos que conducen a la reparación del error.

Como ámbito de investigación, se ha abordado el feedback correctivo escrito desde dos perspectivas teóricas y metodológicas diferentes (Shintani, Ellis y Suzuki, 2014). Primero, en la escritura desde una L2, y otro, desde la adquisición de segundas lenguas (ASL) (Ferris, 2010). Al considerar el feedback desde el área de la escritura como L2, Coyle y Roca de Larios (2014) especifican que el feedback se concibe como un medio para ayudar a los estudiantes a mejorar la calidad y la eficiencia de sus textos, mientras que desde la ASL se observa generalmente como una corrección del error que contribuye al desarrollo lingüístico de los estudiantes.

Desde los planteamientos de una L2 y de la ASL, podemos rescatar un aspecto elemental, que se relaciona con la mejora en la producción escrita de los estudiantes. En ese sentido, al entender FCE como estrategia, es posible en palabras de Salaberry y Altamirano (2001) constituir planes de acción dirigidos al logro de metas de aprendizaje de manera consciente e intencional. Se entiende, también, como la manera en la que el profesor logra que el estudiante note sus errores y se focalice en la forma o estructura gramatical deseada (Sheen, 2011). 
El mejoramiento de los procesos de escritura también es un tema relevante en una lengua materna (LI) porque es un proceso que necesita varias microhabilidades para que resulte de manera exitosa. En ese sentido, Cassany (2014) entrega indicios de cómo el docente de L1 debe corregir a sus estudiantes mediante la focalización de los errores, cómo marcarlos en un texto, qué color de lápiz utilizar, pero esto aún no es suficiente para determinar o combinar estrategias desde este ámbito. Es por ello que el FCE es pertinente, ya que la implementación de estas estrategias es apropiada porque permite al docente apoyar el proceso de enseñanza de la escritura y proporciona al estudiante andamiajes de las formas gramaticales que necesita aprender para dar coherencia y cohesión a los textos, de acuerdo a un contexto comunicativo donde no se memoricen las formas, sino que se modelen a través del aprendizaje de la escritura y la corrección de errores.

El feedback ha tomado diferentes perspectivas, desde la utilización a la forma y control de la lengua y también desde la función que han ejercido los docentes en la corrección de las tareas de escritura para apoyar la precisión lingüística de los estudiantes. En primera instancia en la oralidad, donde el feedback tiene como objetivo apoyar las intervenciones de aprendientes (audiencia real), que interactúan con propósitos concretos dentro de un contexto donde el aprendizaje es considerado una práctica social (Hyland y Hyland, 2006), y, posteriormente, en el ámbito de la escritura y revisión de los textos.

En la dimensión oral, surge la hipótesis de la interacción trabajada por Long (1991), quien en el contexto de la negociación del significado acuña la noción de focalización en la forma (FF), la que atrae la atención de los estudiantes a los elementos lingüísticos que surgen incidentalmente en lecciones en las que el foco está dado por el significado o la comunicación, no por la enseñanza explícita de una forma gramatical.

En relación a este planteamiento, Doughty y Williams (1998) señalan que el objetivo fundamental que tienen los maestros al enseñar una lengua es que esta sea para comunicarse y desarrollar la competencia comunicativa. Por consiguiente, una de las herramientas que se identifican como un instrumento potencial de la focalización en la forma es la corrección de los errores (Ellis, 2005).

En la escritura, de acuerdo a Van Beuningen (2010) en Lillo y Ferreira (2014), se puede señalar que el feedback correctivo escrito puede entenderse mediante dos instancias: la primera, como una metodología reactiva de la FF al centrar la atención del aprendiente en las estructuras lingüísticas en el contexto de realización de una tarea comunicativa de una manera personalizada e individual, mientas que la segunda perspectiva se encuentra en la hipótesis de "noticing”, la que, según Schmidt (2010) en Lillo y Ferreira (2014), sugiere que la atención debe ser dirigida o focalizada en aquellos ámbitos lingüísticos que se quieran desarrollar. De esta forma, el noticing se presenta como un proceso necesario que permite al estudiante atender conscientemente a ciertos rasgos lingüísticos proporcionados en el input y notar la 
brecha que existe entre su IL y la lengua meta. Cabe señalar, según Bleyhl (2009), que, una vez notado este vacío, es posible transformar en intake aquello conscientemente atendido para generar aprendizaje.

El proceso descrito anteriormente se facilita mediante la implementación de estrategias de feedback correctivo escrito, las que permiten focalizar la atención de los estudiantes en formas lingüísticas establecidas para estimular el uso en sus producciones escritas.

\subsection{FCE directo y FCE metalingüístico}

Ellis (2009) propone una clara definición y una ejemplificación de distintos tipos de feedback correctivo escrito. En este trabajo, se utilizó la forma directa y metalingüística con descripción gramatical para entregar FCE a los estudiantes a través de la plataforma Moodle ARCOUdeC.

a. Feedback correctivo directo: El profesor provee al estudiante la forma correcta y se tarja o resalta de alguna forma el error.

Ejemplo: El día de ayer, José Luis Cisternas hombre de 70 años de edad, asistió a la hora que tenía programada en el Hospital de Rancagua, con pesar ya que sentía una molestia, asimismo se encontró con el paro que tenían los funcionarios de dicho Hospital.

Corrección directa (FCE-directo): sin embargo.

b. Feedback correctivo metalingüístico mediante descripción gramatical: El profesor entrega al alumno algún tipo de clave metalingüística con referencia a la naturaleza del error. Por lo tanto, enumera los errores en el texto y escribe (al final de la página) una descripción gramatical para cada error numerado.

Ejemplo: El día de ayer, José Luis Cisternas hombre de 70 años de edad, asistió a la hora que tenía programada en el Hospital de Rancagua, con pesar ya que sentía una molestia, asimismo se encontró con el paro que tenían los funcionarios de dicho Hospital.

Corrección metalingüística (FCE-metalingüístico): Notaste que hay un elemento que cumple una función continuativa que expresa acuerdo. Recuerda que para marcar oposición es necesario utilizar un marcador discursivo con valor continuativo que denote disconformidad.

\subsubsection{Contraste entre el efecto del FCE directo y el FCE metalingüístico}

El FCE ha sido un tema ampliamente estudiado en la enseñanza de segundas lenguas: los estudios de Ferris (1999, 2010) y Truscott (1996, 2008), quienes se cuestionaron la efectividad y contribución del feedback; Ellis (2009), que aportó con una diferenciación de las estrategias de feedback; hasta Razagifard y Razzaghifard (2011), quienes investigaron el impacto de dos tipos de feedback correctivo en el contexto comunicativo mediado por computadora sobre 
el desarrollo del conocimiento del segundo idioma (L2). Finalmente, los últimos trabajo de Bitchner (2017a, 2017b) han abordado una dimensión interesante del feedback al estudiarlo a través de la organización de los géneros académicos. Desde estos autores, podemos destacar la evolución que ha presentado el feedback en sus diferentes procesos y las preguntas que se han realizado estos investigadores en torno al tipo de estrategia y a su efectividad.

Por lo tanto, resulta interesante, entonces, revisar estudios en los que se comparen las estrategias directas en oposición a las metalingüísticas a corto y largo plazo para determinar el efecto en el aprendizaje de formas seleccionadas, los géneros discursivos escritos y los contextos de aplicación.

De acuerdo con lo anterior, el estudio de Shintani, Ellis y Suzuki (2014) comparó los efectos de dos tipos de feedback enfocado en la forma — feedback correctivo directo (FCD) y explicación metalingüística (EM) dada a toda la clase- en la precisión de uso de dos estructuras gramaticales de los estudiantes universitarios japoneses: artículo indefinido y el hipotético condicional; cabe mencionar que ambos tipos de feedback se dieron con y sin la oportunidad de reescribir, pero que la precisión del uso se midió con la escritura de nuevos textos. Como resultado, el feedback que condujo a una mayor precisión a largo plazo para el condicional hipotético fue el feedback con EM, aunque esta estrategia no fue tan eficaz para el artículo indefinido.

Otro estudio que se focalizó en los efectos de la corrección inmediata y tardía fue el que realizaron Li, Zhu y Ellis (2016) mediante la escritura de narraciones en las que se elicitaba el uso de la construcción pasiva en inglés. La investigación contó con tres grupos experimentales y los principales resultados evidenciaron que el feedback solo ayudó al desarrollo del conocimiento declarativo/explícito y que la ventaja encontrada para la retroalimentación inmediata se debió a que los estudiantes usaron la retroalimentación progresivamente en la producción de nuevas oraciones pasivas pasadas, mientras que esto no ocurrió en la condición de feedback tardío.

En Chile, también se han realizado investigaciones en torno al feedback correctivo escrito, las que tampoco han sido contundentes en torno a la estrategia ni a la forma utilizada. En ese sentido, Ferreira $(2015,2017)$ apunta a que la estrategia metalingüística es más efectiva a corto y largo plazo en aprendientes de español como lengua extranjera cuya lengua de origen varía entre inglés, francés, alemán e italiano.

Otro estudio encabezado por Ortiz y Ferreira (2014) también indica que la corrección metalingüística de tipo indirecta presenta mejores resultados a corto y largo plazo en estudiantes de inglés como lengua extranjera en la escritura de ensayos colaborativos. En ese sentido, el trabajo de Muñoz y Ferreira (2017) estableció que estas estrategias de tipo indirecto también podían ser efectivas en otro contexto de aprendizaje, pues su investigación se centra en estudiantes de octavo año básico provenientes de un colegio particular pagado cuya lengua de origen es el español. 
De acuerdo con estas investigaciones, se puede extrapolar que el feedback es un tema plenamente estudiado y que aún no hay una respuesta única, sino indicios probatorios en cuanto a metodología y resultados, de acuerdo con diferentes variantes, tales como: contexto de aplicación, competencia de los estudiantes y el género discursivo que ellos escriban, pues este elemento condiciona de modo natural la elicitación de una determinada forma lingüística.

\subsection{Forma lingüística a nivel macroestructural: marcadores discursivos con valor organizativo}

De acuerdo con el trabajo de Martín Zorraquino y Portolés Lázaro (1999), los marcadores discursivos son unidades lingüísticas invariables. Según Silva Corvalán (2001: 218), "no poseen la capacidad de flexionarse, debido a la pérdida de su contenido semántico por los procesos de gramaticalización”, por lo que no ejercen una función sintáctica en el marco de la predicación oracional (son elementos marginales) y guían las inferencias que se realizan en la comunicación de acuerdo con las propiedades morfosintácticas, semánticas y pragmáticas. En ese sentido, Murillo (2000) plantea que los marcadores tienen un significado en el procesamiento, pues ese significado expresa una relación conceptual con las unidades que lo han originado.

Los marcadores, generalmente, aparecen clasificados en cinco categorías (Portolés, 2014): estructuradores de la información, conectores, reformuladores, marcadores conversacionales y operadores. Por lo tanto, aportan a la estructura informativa del discurso, añadiendo, contraponiendo y rectificando informaciones.

Asimismo, según la perspectiva de Van Dijk (1980), los marcadores son los elementos encargados de organizar la información contenida en el texto y, en consecuencia, forman parte de la "macroestructura".

A partir del punto de vista expuesto, y según los planteamientos de Pottier (1993), es posible considerar el proceso de construcción del discurso como un continuo compuesto por un inicio, una continuación y un cierre. Así, los marcadores discursivos se clasifican de acuerdo con su valor en tres grupos, según Pons (1998) y Errázuriz (2012):

1. Marcadores con valor de inicio: Manifiestan una visión prospectiva y de apertura hacia el acto enunciativo que introducen; controlan el comienzo de un acto discursivo; corresponden a enlaces con valor de topicalización (introducen un tópico). Por ejemplo: "Para comenzar, la situación de los implicados".

2. Marcadores con valor de continuación: Señalan el proceso de evolución del acto comunicativo; infieren que se enunció algo anteriormente; permiten que el hablante sea capaz de guiar o cambiar su acto comunicativo al amplificar, ordenar, manifestar acuerdo (al reformular, ejemplificar y explicar) o desacuerdo. En esta categoría se encuentran los enlaces de adición, continuidad, orden (enumeración y distribución), conformidad (equiva- 
lencia, inclusión, causalidad) y disconformidad (oposición). Por ejemplo: "El sujeto falleció en el lugar. Sin embargo, aún no existen pruebas".

3. Marcadores con valor de cierre: Manifiestan una visión retrospectiva de la información ya expresada; pueden indicar recapitulación, reformulación o cierre de lo anteriormente afirmado; usualmente, presentan una perspectiva de epílogo, conclusión o resumen. Por ejemplo: "En resumen, se espera que la verdad...".

Pons (1998) y Errázuriz (2012) clasifican los marcadores de acuerdo a estos tres momentos que suelen darse en la configuración textual, los que permiten estructurar la superestructura textual y entender el texto en su estructura cronológica secuencial (crónica informativa) y a nivel macroestructural; ya en esta dimensión los marcadores discursivos son útiles porque guían el proceso inferencial y proporcionan información a nivel de la organización y estructuración semántica.

\section{Metodología}

Con el propósito de determinar la efectividad de las estrategias de FCE directo y claves metalingüísticas a través de descripciones gramaticales (Ellis, 2009) en el mejoramiento de errores focalizados en crónicas periodísticas, se llevó a cabo un estudio experimental de feedback correctivo escrito (FCE) con dos grupos experimentales y uno control, compuestos por estudiantes de periodismo de primer año de universidad.

\subsection{Preguntas de investigación}

La pregunta de investigación que orienta este estudio es:

1. ¿Qué estrategia de feedback correctivo escrito es más efectiva para disminuir errores en el uso de marcadores discursivos con valor organizativo en la escritura de crónicas periodísticas?

\subsection{Hipótesis}

$\mathrm{Hi}_{1} \mathrm{El}$ feedback correctivo escrito directo representa una estrategia que favorece el uso preciso de marcadores discursivos con valor organizativo en la escritura de crónicas periodísticas.

$\mathrm{Hi}_{01} \mathrm{El}$ feedback correctivo escrito directo representa una estrategia que no favorece el uso preciso de marcadores discursivos con valor organizativo en la escritura de crónicas periodísticas.

$\mathrm{Hi}_{2}$ El feedback correctivo escrito metalingüístico con descripción gramatical representa una estrategia que favorece el uso preciso de marcadores discursivos con valor organizativo en la escritura de crónicas periodísticas. 
$\mathrm{H}_{02}$ El feedback correctivo escrito metalingüístico con descripción gramatical no representa una estrategia que favorece el uso preciso de marcadores discursivos con valor organizativo en la escritura de crónicas periodísticas.

$\mathrm{Hi}_{3}$ El grupo control que no recibe feedback correctivo escrito, sino comentarios generales utiliza de modo preciso marcadores discursivos con valor organizativo en la escritura de crónicas periodísticas.

$\mathrm{H}_{03}$ El grupo control que no recibe feedback correctivo escrito, sino comentarios generales no utiliza de modo preciso marcadores discursivos con valor organizativo en la escritura de crónicas periodísticas.

\subsection{Diseño}

Este estudio presenta un diseño experimental, pretest, post-test inmediato y post-test diferido. Una de las fortalezas de este diseño es la forma en que se mide la adquisición de las estructuras, debido a que se incorpora una medida longitudinal de mejoramiento de la precisión gramatical por medio de un pretest, post-test inmediato y post-test diferido (Bitchener, 2008).

El post-test inmediato se administra para minimizar los posibles efectos de otras variables intervinientes una vez realizado el tratamiento, mientras que el post-test diferido es usado para medir la retención de las estructuras en el tiempo, y aunque no es posible eliminar los efectos de las variables intervinientes entre el post-test inmediato y el diferido, de todas formas su utilización es una medida válida del nivel de retención observado en el post-test inmediato. En estas mediciones del aprendizaje, el tiempo de desfase de la aplicación de los post-test inmediatos corresponde a uno o dos días después del tratamiento (Lillo, 2014; Lillo y Sáez, 2017). Por otra parte, dado que varios estudios han reportado efectos en post-test diferidos, pero no en post-test inmediatos, la investigación sugiere la utilización de ambos tipos de post-test para estimar el efecto en el aprendizaje (Loewen, 2012).

\subsection{Participantes}

La población de estudiantes de periodismo de primer año corresponde a 52 sujetos; sin embargo, para efectos de medición, se tomó como muestra a los sujetos de acuerdo con los siguientes criterios:

- Estudiantes de primer año de ingreso.

- La forma de recoger la información fue voluntaria.

Es por ello que los grupos quedaron compuestos de 13 sujetos cada uno. La selección fue aleatoria y se identificó a tres grupos: A, B y C. 
La muestra corresponde a 39 sujetos que estudian periodismo y se estructura de la siguiente manera:

- Grupo control: 13 estudiantes (GC).

- Grupo experimental 1: 13 estudiantes (GE1).

- Grupo experimental 2: 13 estudiantes GE2).

Los 39 sujetos, cuyas edades fluctúan entre 18 y 21 años, cursan la asignatura Producción de Textos, impartida el primer semestre por el Departamento de Comunicación Social de una universidad chilena. EI GC no recibió feedback, solo comentarios como "bien", "buen trabajo", "debes mejorar", mientras que el GEI recibió feedback correctivo directo, donde se proveyó explícitamente la forma correcta del marcador que necesitaba, por ejemplo, "allí debió usar sin embargo, no además"; finalmente, el GE2 recibió estrategias de feedback metalingüísticas mediante descripciones gramaticales, por ejemplo, "notó que usó un marcador que expresa continuidad, sin embargo usted quiere comenzar el párrafo, por lo tanto necesita un marcador con función topicalizadora”.

\subsection{Descripción de los instrumentos: pretest, post-test inmediato y post-test diferido}

Los instrumentos y las tareas se implementaron en la plataforma ARCOUdeC, que a través del sistema de gestión de aprendizaje Moodle permite a los docentes estructurar tareas de escritura y ejercicios de práctica para optimizar el desarrollo de sus clases. En este contexto, la docente encargada de la asignatura utilizó esta plataforma para implementar un diseño de tareas de escritura con la finalidad de que los estudiantes redactaran semanalmente crónicas periodísticas. Se trabajó con este género periodístico porque permite, según su textura discursiva de tipo narrativo-descriptiva, elicitar el uso de marcadores discursivos con valor organizativo; además, es una actividad que realizan con frecuencia los estudiantes de primer año de la carrera de Periodismo y que necesitan manejar, de acuerdo a los programas de estudio.

Las crónicas informativas eran corregidas mediante estrategias de feedback correctivo por la docente a cargo de la asignatura y por dos evaluadores externos ayudantes de la asignatura, que cursaban quinto año de Periodismo. Los tres evaluadores se reunían todas las semanas y revisan en conjunto todas las correcciones que se entregarían a los estudiantes con la finalidad de aunar criterios.

Cada uno de los test se construyó basado en los principios metodológicos del enfoque por tareas, por lo que las tareas de escritura se elaboraron con contenidos auténticos y en un contexto comunicativo (Estaire, 2007). El pretest consistió en la escritura de una crónica periodística de 350 palabras, respecto al caso de un senador de la República de Chile, acusa- 
do de cohecho. Este caso fue denominado por la prensa como caso Longueira. Para ello, los estudiantes ingresaron a la plataforma Moodle en la que se encontraba la actividad, leyeron las instrucciones y escribieron su texto. Esta tarea favoreció la elicitación de las estructuras lingüísticas (MDO) según el objetivo del estudio.

En relación con el post-test inmediato, en este se elaboró una crónica informativa de 350 palabras sobre la salud pública en Chile, que se aplicó en la sexta semana con el objetivo de evaluar el aprendizaje de los MDO utilizando estrategias de FCE para medir a corto plazo la efectividad de las estrategias de FCE utilizadas.

Para concluir la intervención lingüística, en la décima semana, se aplicó el post-test diferido con la finalidad de evaluar la retención a largo plazo y la transferencia de los nuevos conocimientos proporcionados durante las diferentes intervenciones del tratamiento. En este caso, los estudiantes redactaron una crónica de 350 palabras, sobre los movimientos y marchas estudiantiles.

Los tres instrumentos aplicados fueron similares en el formato y en el medio en que fueron realizados, es decir, los tres correspondían a la escritura de crónicas periodísticas informativas, producidas por estudiantes de periodismo en la plataforma Moodle ARCOUdeC en un laboratorio de computación; la única diferencia es el momento de la aplicación.

El tiempo que tuvieron los estudiantes para realizar cada una de las tres pruebas (pretest, post-test y post-test diferido) fue de 90 minutos.

La intervención lingüística siguió el modelo del proyecto FONDECYT n. ${ }^{0} 1140651$ propicio para estudiar estrategias de FCE con pretest, post-test inmediato y post-test diferido con 10 semanas de duracióna través de una intervención lingüística.

\section{TABLA 1}

Instrumentos de medición

\begin{tabular}{llll}
$\begin{array}{l}\text { MOMENTOS DE } \\
\text { EVALUACIÓN }\end{array}$ & PRETEST & POST-TEST & POST-TEST DIFERIDO \\
Semana & 1 & 6 & 10 \\
\hline Tema & Caso Longueira & Salud pública en Chile & Movimientos estudiantiles \\
\hline Tarea & $\begin{array}{l}\text { Escritura de una } \\
\text { crónica informativa }\end{array}$ & $\begin{array}{l}\text { Escritura de una } \\
\text { crónica informativa }\end{array}$ & $\begin{array}{l}\text { Escritura de una } \\
\text { crónica informativa }\end{array}$ \\
\hline Extensión & 350 palabras & 350 palabras & 350 palabras \\
\hline Tiempo & 90 minutos & 90 minutos & 90 minutos \\
\hline Lugar & Laboratorio de & Laboratorio de & Laboratorio de \\
& computación & computación & computación \\
\hline
\end{tabular}




\subsection{Las tareas de tratamiento}

Después del pretest se inició el proceso de tratamiento por medio de 4 sesiones. En la tabla 2 se presenta la secuencia de cada una de las sesiones de escritura; la primera consistía en proporcionar el contexto para desarrollar la tarea, luego un andamiaje gramatical, posteriormente la tarea final y, por último, el cierre metacognitivo. Las tareas de tratamiento fueron iguales para los tres grupos.

\section{TABLA 2}

Secuencia de la clase para elicitar la tarea de escritura

\begin{tabular}{|c|c|c|c|c|}
\hline SEMANA & $\begin{array}{l}\text { PRETAREA } \\
\text { (INICIO) }\end{array}$ & TAREAS & TAREA FINAL & CIERRE \\
\hline $2,3,4,5$ & $\begin{array}{l}\text { Contexto temático, } \\
\text { según el tópico } \\
\text { de la tarea }\end{array}$ & $\begin{array}{l}\text { Andamiaje gramatical } \\
\text { (tareas de completación, } \\
\text { términos pareados, } \\
\text { preguntas de desarrollo } \\
\text { y de aplicación) }\end{array}$ & $\begin{array}{l}\text { Escritura de } \\
\text { una crónica } \\
\text { informativa (cuatro } \\
\text { crónicas en total, } \\
\text { una cada semana) }\end{array}$ & $\begin{array}{l}\text { Reflexión } \\
\text { metacognitiva } \\
\text { (reflexión sobre la } \\
\text { tareas realizadas) }\end{array}$ \\
\hline
\end{tabular}

La tabla 2 presenta una secuencia de clases que se estructura mediante el enfoque por tareas, según Estaire (2007). La clase se dividía en cuatro procesos. Primero se muestra la pretarea, es decir, el contexto respecto del tema que se va a tratar en la clase, y el docente presenta los contenidos. Posteriormente, en la segunda fase, se presentan las tareas; estas corresponden a tareas de escritura que se inician con ejercicios de manejo de la lengua, mediante el cual la docente provee un andamiaje gramatical; ellas se dividen en: preguntas de aplicación (tarea 1), ejercicios de completación (tarea 2), términos pareados (tarea 3) y preguntas abiertas (tarea 4) sobre marcadores del discurso. Luego, en la tercera fase, la tarea final de la clase corresponde a la escritura de una crónica informativa. Finalmente, en la cuarta fase, se presenta el cierre de la clase, que concierne a una reflexión metacognitiva. Estas tareas corresponden a la intervención lingüística con tareas idénticas para los tres grupos que se realizó luego de la aplicación del pretest (semana 1) y hasta la aplicación del post-test (semana 6).

La tarea final de la clase correspondía a la escritura de una crónica informativa, con una estructura predeterminada: epígrafe, titular, bajada, lead y cuerpo (definida y explicada en la plataforma ARCOUdeC). La extensión era de 350 palabras y los temas de cada crónica se especificaban antes de la sesión. Esto para que los sujetos estudiaran el tema, pues no tenían acceso a buscar información durante la tarea.

Una vez que los estudiantes escribían su crónica, la docente responsable corregía los textos y luego se reunía con los dos ayudantes para aunar criterios sobre la corrección pro- 
porcionada a los estudiantes, de acuerdo a las estrategias elegidas para desarrollar esta intervención. El procedimiento para entregar el FC escrito para cada uno de los grupos experimentales fue el siguiente:

a. La docente especialista corrigió todos los textos del grupo experimental 1 y 2. Además entregó comentarios generales al grupo control, tales como: ¡Debes mejorar la redacción!, iSu escrito presenta problemas de legibilidad!, iAvanza bastante bien en el trabajo! Luego, con los dos ayudantes revisaban nuevamente todos los textos y las correcciones que verían los estudiantes en la plataforma ARCOUdeC.

b. El día anterior a la nueva escritura de la crónica, se liberaba la corrección en la plataforma Moodle ARCOUdeC y los alumnos la revisaban y anotaban sus dudas en el foro de la asignatura. La revisión del feedback era obligatoria para todos los estudiantes, pues la plataforma permitía registrar las visitas y el tiempo de conexión.

c. Al día siguiente, los estudiantes comenzaban la siguiente tarea y no podían acceder nuevamente al feedback.

d. Este proceso se repitió durante las 4 sesiones que recibieron tratamiento, pues los estudiantes escribieron textos nuevos en cada clase.

\section{Resultados y análisis}

El experimento consideró una variable independiente y una dependiente; la variable independiente corresponde a cada uno de los dos grupos experimentales (EI, feedback correctivo directo, y E2, feedback metalingüístico); la variable dependiente corresponde a la diferencia en la disminución de los errores focalizados en este estudio, es decir, MDO, al comparar el pretest y los post-test inmediato y diferido.

Los puntajes obtenidos a partir de la contabilización de errores fueron procesados y analizados con el programa Statistical Package for the Social Sciences 22 (SPSS). Este programa permite examinar tanto las distribuciones de frecuencia para cada variable en particular como las relaciones entre ellas. Los modelos estadísticos más pertinentes para este estudio corresponden a las pruebas paramétricas ANOVA de un factor. Una vez aplicada esta prueba, se utilizó una prueba T de Student para muestras independientes.

\subsection{Análisis de resultados en el uso de MDO}

Para analizar la presencia de los marcadores discursivos en las crónicas se utilizó como medida de análisis la contabilización de los errores en las crónicas escritas por los estudiantes. Esta medida examina el grado de precisión con que los estudiantes utilizan la forma lingüística seleccionada, es decir, marcadores discursivos con valor organizativo. 
La tabla 3 presenta el número total de errores en el uso de marcadores discursivos organizativos (MDO) utilizados en la escritura de las crónicas en el pretest, post-test inmediato y post-test diferido.

\section{TABLA 3}

Total de errores de la forma lingüística utilizada en el estudio: pretest, post-test y post-test diferido

\begin{tabular}{lllllllllllll}
$\begin{array}{l}\text { MOMENTOS } \\
\text { EVALUACIÓN }\end{array}$ & PRETEST & \multicolumn{1}{c}{ POST-TEST INMEDIATO } & \multicolumn{3}{c}{ POST-TEST DIFERIDO } \\
Grupos & GC & GE1 & GE2 & total & GC & GE1 & GE2 & total & GC & GE1 & GE2 & total \\
\hline Erróneas & 25 & 23 & 41 & $\mathbf{8 9}$ & 32 & 27 & 9 & $\mathbf{6 8}$ & 14 & 24 & 3 & $\mathbf{4 1}$ \\
\hline Total \% & $\mathbf{2 8 , 1}$ & $\mathbf{2 5 , 8}$ & $\mathbf{4 6 , 1}$ & $\mathbf{1 0 0}$ & $\mathbf{4 7 , 1}$ & $\mathbf{3 9 , 7}$ & $\mathbf{1 3 , 2}$ & $\mathbf{1 0 0}$ & $\mathbf{3 4 , 2}$ & $\mathbf{5 8 , 5}$ & $\mathbf{7 , 3}$ & $\mathbf{1 0 0}$ \\
\hline
\end{tabular}

En la tabla 3 se presentan los errores en el uso de marcadores discursivos con valor organizativo (MDO) en los tres momentos en que se midió la intervención lingüística, según tres pruebas: pretest, post-test inmediato y post test-diferido.

La tabla, en la segunda fila, muestra los grupos de la intervención: grupo control, que no recibió feedback; el grupo experimental 1, que recibió feedback directo, y el grupo experimental 2, que recibió feedback mediante claves metalingüísticas.

El total de errores de los 3 grupos en el pretest corresponde a 89, mientras que en la segunda medición los errores disminuyen a 68 y, en la última medición, los errores disminuyen aún más a un total de 41. Esto se debe a que el grupo experimental 2 disminuyó sus errores en el uso de MDO.

Los errores cometidos por el GE2 disminuyeron de 41 en primera instancia a 9 en el posttest, para finalizar la intervención cometiendo solo 3 errores. En ese sentido, estos resultados se relacionan con los encontrados por Ortiz y Ferreira (2014), Ferreira (2017) y Muñoz y Ferreira (2017), que indican que la corrección implícita es adecuada para que el estudiante note y repare su error. Por lo tanto, la estrategia metalingüística especifica la importancia de la reflexión dentro de la corrección, lo que hace que la reparación de errores se mantenga a largo plazo.

\subsubsection{Normalización de los datos}

Una vez contabilizados los usos de MDO, se trabajó solo con los errores que cometieron los estudiantes en las tres mediciones. Sin embargo, para efectos de la normalización de los datos se estableció un rango de valores que posteriormente se introdujeron al software estadístico SPSS: 


\section{TABLA 4}

Pruebas de normalidad

\begin{tabular}{|c|c|c|c|c|c|c|c|}
\hline \multirow{2}{*}{\multicolumn{2}{|c|}{$\begin{array}{l}\text { GRUPOS ESTADÍSTICOS } \\
\text { DE ESTUDIOS }\end{array}$}} & \multicolumn{3}{|c|}{ KOLMOGOROV-SMIRNOV ${ }^{\mathrm{a}}$} & \multicolumn{3}{|c|}{ SHAPIRO-WILK } \\
\hline & & ESTADISTICO & GL & SIG & ESTADÍSTICO & GL & SIG \\
\hline \multirow[t]{3}{*}{ Pretest } & Sujeto Grupo M & 189 & 13 &, $200^{*}$ & 903 & 13 & ,147 \\
\hline & Sujeto Grupo D & 261 & 13 & 015 & 853 & 13 & 031 \\
\hline & Sujeto Grupo C & 307 & 13 & 001 & 762 & 13 & 002 \\
\hline
\end{tabular}

Según la prueba de normalidad KS se distribuyen normalmente los datos, por lo tanto hay que afirmar el supuesto de normalidad en los grupos control — grupo C - experimental 1 - grupo D-y experimental 2 - grupo $\mathrm{M}$ - (estadísticos en .189 y .307; gl: 13; p>0.5).

El segundo supuesto de normalidad corresponde a la independencia de las observaciones; en este punto se puede sostener que las personas que componen los grupos son distintas. Luego, en el tercer supuesto, respecto a la equivalencia de grupos, los tamaños de las muestras son iguales en cada grupo; es un indicio de que hay equivalencia de grupos en las poblaciones. En ese sentido, la prueba de independencia de las observaciones y la de equivalencia se comprueban.

\subsubsection{Prueba de homogeneidad de varianzas}

Una vez normalizados los datos, se aplicó la prueba de homogeneidad de varianzas para el pretest.

\section{TABLA 5}

Prueba de homogeneidad de varianzas

\begin{tabular}{cccc} 
ESTADISTICO DE LEVENE & GL1 & GL2 & SIG. \\
\hline 125 & 2 & 36 &, 883 \\
\hline
\end{tabular}

La prueba de homogeneidad de la varianza LEVENE indica que se cumple el supuesto (estadístico .125; glu: 2, y g|2: 36; p> a .05).

\subsubsection{Análisis de varianza de un factor}

Para comparar el promedio de los tres grupos que componen el experimento, se utilizó ANOVA de un factor con la finalidad de comprobar la diferencia entre los promedios de los grupos comparados. 


\section{TABLA 6}

ANOVA de un factor para las tres mediciones

\begin{tabular}{ccccccc} 
& SUMA DE & GL & $\begin{array}{c}\text { MEDIA } \\
\text { CUADRADOS }\end{array}$ & & F & SIG. \\
CUADRATICA & & \\
Pretest & Intergrupos & 14,974 & 2 & 7,487 & 4,42 & \multirow{2}{*}{019} \\
& Intragrupos & 6,923 & 36 & 1,692 & & \\
& Total & 75,897 & 38 & & & \\
\hline Post-test & Intergrupos & 22,513 & 2 & 11,256 & 7,958 & \multirow{2}{*}{001} \\
& Intragrupos & 50,923 & 36 & 1,415 & & \\
& Total & 73,436 & 38 & & & \multirow{2}{*}{000} \\
\hline Post-test & Intergrupos & 16,974 & 2 & 8,487 & 10,564 & \\
diferido & Intragrupos & 28,923 & 36 &, 803 & & \\
& Total & 45,897 & 38 & & &
\end{tabular}

Sobre la prueba de ANOVA unifactorial, como la hipótesis de investigación es que hay diferencia, entonces la hipótesis nula es que no hay diferencia entre grupos. La significancia está sobre .05 (p<.019) en el pretest, lo que indica que antes de la intervención lingüística-tratamiento no hay diferencias. Sin embargo, en el post-test inmediato y post-test diferido, sí hay diferencias entre grupos, por efecto del tratamiento.

Por lo tanto, se rechaza la hipótesis nula y se acepta la hipótesis de investigación, es decir, que hay diferencia entre los grupos para el post-test inmediato ( $F(7.958)$; $p$ <.001) y el post-test diferido (F(10,564); $p<.000)$.

Para determinar entre qué grupos se genera esa diferencia, entonces se aplicó la prueba post hoc de Tukey, que muestra las diferencias significativas que hay por grupo.

\section{TABLA 7}

Comparaciones múltiples

\begin{tabular}{|c|c|c|c|c|c|c|c|}
\hline \multicolumn{3}{|c|}{ HSD DE TUKEY } & \multirow[t]{2}{*}{$\begin{array}{l}\text { DIFERENCIAS } \\
\text { DE MEDIAS (I-J) }\end{array}$} & \multirow[t]{2}{*}{$\begin{array}{l}\text { ERROR } \\
\text { TÍPICO }\end{array}$} & \multirow[t]{2}{*}{ SIG. } & \multicolumn{2}{|c|}{$\begin{array}{l}\text { INTERVALO DE } \\
\text { CONFIANZA AL 95\% }\end{array}$} \\
\hline $\begin{array}{l}\text { VARIABLE } \\
\text { DEPENDIENTE }\end{array}$ & $\begin{array}{l}\text { (I) GRUPOS } \\
\text { (J) GRUPOS }\end{array}$ & $\begin{array}{l}\text { EESTUDIO (TRES) } \\
\text { EESTUDIO (TRES) }\end{array}$ & & & & $\begin{array}{l}\text { LÍMITE } \\
\text { INFERIOR }\end{array}$ & $\begin{array}{l}\text { LÍMITE } \\
\text { SUPERIOR }\end{array}$ \\
\hline \multirow[t]{3}{*}{ Pretest } & $\begin{array}{l}\text { Sujeto } \\
\text { Grupo M }\end{array}$ & $\begin{array}{l}\text { Sujeto grupo D } \\
\text { Sujeto grupo C }\end{array}$ & $\begin{array}{l}1,385^{*} \\
1,231\end{array}$ & $\begin{array}{l}, 510 \\
, 510\end{array}$ & $\begin{array}{l}, 027 \\
, 054\end{array}$ & $\begin{array}{l}, 14 \\
-, 02\end{array}$ & $\begin{array}{l}2,63 \\
2,48\end{array}$ \\
\hline & $\begin{array}{l}\text { Sujeto } \\
\text { Grupo D }\end{array}$ & $\begin{array}{l}\text { Sujeto grupo M } \\
\text { Sujeto grupo C }\end{array}$ & $\begin{array}{l}-1,385^{\star} \\
-, 154\end{array}$ & $\begin{array}{l}, 510 \\
, 510\end{array}$ & $\begin{array}{l}, 027 \\
, 951\end{array}$ & $\begin{array}{l}-2,63 \\
-1,40\end{array}$ & $\begin{array}{l}-, 14 \\
1,09\end{array}$ \\
\hline & $\begin{array}{l}\text { Sujeto } \\
\text { Grupo C }\end{array}$ & $\begin{array}{l}\text { Sujeto grupo M } \\
\text { Sujeto grupo D }\end{array}$ & $\begin{array}{l}-1,231 \\
, 154\end{array}$ & $\begin{array}{l}, 510 \\
, 510\end{array}$ & $\begin{array}{l}, 054 \\
, 951\end{array}$ & $\begin{array}{l}-2,48 \\
-1,09\end{array}$ & $\begin{array}{l}, 02 \\
1,40\end{array}$ \\
\hline
\end{tabular}




\begin{tabular}{|c|c|c|c|c|c|c|c|}
\hline \multirow[t]{3}{*}{ Post-test } & $\begin{array}{l}\text { Sujeto } \\
\text { Grupo M }\end{array}$ & $\begin{array}{l}\text { Sujeto grupo D } \\
\text { Sujeto grupo C }\end{array}$ & $\begin{array}{l}-1,385^{*} \\
-1,769^{*}\end{array}$ & $\begin{array}{l}, 466 \\
, 466\end{array}$ & $\begin{array}{l}, 014 \\
, 002\end{array}$ & $\begin{array}{l}-2,52 \\
-2,91\end{array}$ & $\begin{array}{l}-, 24 \\
-, 63\end{array}$ \\
\hline & $\begin{array}{l}\text { Sujeto } \\
\text { Grupo D }\end{array}$ & $\begin{array}{l}\text { Sujeto grupo M } \\
\text { Sujeto grupo C }\end{array}$ & $\begin{array}{l}1,385^{*} \\
-, 385\end{array}$ & $\begin{array}{l}, 466 \\
, 466\end{array}$ & $\begin{array}{l}, 014 \\
, 690\end{array}$ & $\begin{array}{c}, 24 \\
-1,52\end{array}$ & $\begin{array}{l}2,52 \\
, 76\end{array}$ \\
\hline & $\begin{array}{l}\text { Sujeto } \\
\text { Grupo C }\end{array}$ & $\begin{array}{l}\text { Sujeto grupo M } \\
\text { Sujeto grupo D }\end{array}$ & $\begin{array}{c}1,769^{*} \\
, 385\end{array}$ & $\begin{array}{l}, 466 \\
, 466\end{array}$ & $\begin{array}{l}, 002 \\
, 690\end{array}$ & $\begin{array}{l}, 63 \\
-, 76\end{array}$ & $\begin{array}{l}2,91 \\
1,52\end{array}$ \\
\hline \multirow[t]{3}{*}{$\begin{array}{l}\text { Post-test } \\
\text { diferido }\end{array}$} & $\begin{array}{l}\text { Sujeto } \\
\text { Grupo M }\end{array}$ & $\begin{array}{l}\text { Sujeto grupo D } \\
\text { Sujeto grupo C }\end{array}$ & $\begin{array}{l}-1,615^{\star} \\
-, 846\end{array}$ & $\begin{array}{l}, 352 \\
, 352\end{array}$ & $\begin{array}{l}, 000 \\
, 054\end{array}$ & $\begin{array}{l}-2,47 \\
-1,71\end{array}$ & $\begin{array}{l}-, 76 \\
, 01\end{array}$ \\
\hline & $\begin{array}{l}\text { Sujeto } \\
\text { Grupo D }\end{array}$ & $\begin{array}{l}\text { Sujeto grupo M } \\
\text { Sujeto grupo C }\end{array}$ & $\begin{array}{l}1,615^{*} \\
, 769\end{array}$ & $\begin{array}{l}, 352 \\
, 352\end{array}$ & $\begin{array}{l}, 000 \\
, 087\end{array}$ & $\begin{array}{l}, 76 \\
-0,9\end{array}$ & $\begin{array}{l}2,47 \\
1,63\end{array}$ \\
\hline & $\begin{array}{l}\text { Sujeto } \\
\text { Grupo C }\end{array}$ & $\begin{array}{l}\text { Sujeto grupo M } \\
\text { Sujeto grupo D }\end{array}$ & $\begin{array}{l}, 846 \\
-, 769\end{array}$ & $\begin{array}{l}, 352 \\
, 352\end{array}$ & $\begin{array}{l}, 054 \\
, 087\end{array}$ & $\begin{array}{l}-, 01 \\
-1,63\end{array}$ & $\begin{array}{l}1,71 \\
, 09\end{array}$ \\
\hline
\end{tabular}

De acuerdo con la prueba post hoc de Tukey, se observa que las diferencias significativas se encuentran en el grupo metalingüístico (grupo M), que se presenta con significancia .027 en el pretest, 0.14 en el post-test inmediato y 0.00 en el post-test diferido. Por lo tanto, hay una diferencia con el grupo directo (grupo D) .027 en relación al grupo metalingüístico (p<.05).

Se observa que las diferencias se encuentran entre los grupos que recibieron y no recibieron tratamiento. Sin embargo, la diferencia con significancia se da solo en el grupo metalingüístico ( $p<.05)$.

\subsubsection{Prueba T para muestras independientes}

Una vez evaluado el cumplimiento de los supuestos anteriores, en relación a la normalidad de los datos, se procede a llevar a cabo la Prueba T para muestras independientes.

\section{TABLA 8}

Tabla con los estadísticos descriptivos de cada grupo

\begin{tabular}{|c|c|c|c|c|c|}
\hline \multicolumn{6}{|c|}{ ESTADÍSTICOS DE GRUPO } \\
\hline & $\begin{array}{c}\text { GRUPOS DE } \\
\text { ESTUDIOS (TRES) }\end{array}$ & $\mathbf{N}$ & MEDIA & $\begin{array}{l}\text { DESVIACIÓN } \\
\text { TíP. }\end{array}$ & $\begin{array}{l}\text { ERROR TÍP. } \\
\text { DE LA MEDIA }\end{array}$ \\
\hline \multirow[t]{2}{*}{ Pretest } & Sujeto Grupo M & 13 & 3,15 & 1,345 & 373 \\
\hline & Sujeto Grupo D & 13 & 1,77 & 1,301 &, 361 \\
\hline \multirow[t]{2}{*}{ Post-test } & Sujeto Grupo M & 13 & ,69 & 1,032 & ,286 \\
\hline & Sujeto Grupo D & 13 & 2,08 & 1,115 & 309 \\
\hline \multirow{2}{*}{$\begin{array}{l}\text { Post-test } \\
\text { diferido }\end{array}$} & Sujeto Grupo M & 13 &, 23 &, 439 &, 122 \\
\hline & Sujeto Grupo D & 13 & 1,85 & 1,214 &, 337 \\
\hline
\end{tabular}


La tabla 8 presenta los estadísticos descriptivos para cada uno de los grupos que se están comparando. En esta tabla, se analizó si los promedios de los grupos se ajustan a lo estipulado por la hipótesis de investigación.

En este caso, los promedios de los dos grupos resultaron consistentes con la hipótesis de investigación, es decir, hay diferencias. Por lo tanto, se procede a analizar los resultados de la prueba T de Student para determinar la significación estadística de la diferencia entre dichos promedios.

\section{TABLA 9}

Prueba de muestras independientes

\begin{tabular}{|c|c|c|c|c|c|c|c|c|}
\hline & & \multicolumn{2}{|c|}{$\begin{array}{c}\text { PRUEBA DE LEVENE } \\
\text { PARA LA IGUALDAD } \\
\text { DE VARIANZAS }\end{array}$} & \multicolumn{5}{|c|}{ PRUEBA T PARA LA IGUALDAD DE MEDIAS } \\
\hline & & $\mathbf{F}$ & SIG & $\mathbf{T}$ & GL & $\begin{array}{l}\text { SIG. } \\
\text { (BIL.) }\end{array}$ & $\begin{array}{c}\text { DIF. ENTRE } \\
\text { MEDIAS }\end{array}$ & $\begin{array}{l}\text { ERROR TÍP. } \\
\text { DE LA DIF. }\end{array}$ \\
\hline $\begin{array}{l}\text { Pre } \\
\text { test }\end{array}$ & $\begin{array}{l}\text { Se han } \\
\text { asumido dif. } \\
\text { No se han } \\
\text { asumido dif. }\end{array}$ & 198 & 661 & $\begin{array}{l}2,668 \\
2,668\end{array}$ & $\begin{array}{c}24 \\
23,974\end{array}$ & $\begin{array}{l}, 013 \\
, 013\end{array}$ & $\begin{array}{l}1,385 \\
1,385\end{array}$ & $\begin{array}{l}.519 \\
.519\end{array}$ \\
\hline $\begin{array}{l}\text { Post } \\
\text { test }\end{array}$ & $\begin{array}{l}\text { Se han } \\
\text { asumido dif. } \\
\text { No se han } \\
\text { asumido dif. }\end{array}$ & ,194 &, 663 & $\begin{array}{l}-3,286 \\
-3,286\end{array}$ & $\begin{array}{c}24 \\
23,856\end{array}$ & $\begin{array}{l}, 003 \\
, 003\end{array}$ & $\begin{array}{l}-1,385 \\
-1,385\end{array}$ &, 421 \\
\hline $\begin{array}{l}\text { Post } \\
\text { test } \\
\text { Dif }\end{array}$ & $\begin{array}{l}\text { Se han } \\
\text { asumido dif. } \\
\text { No se han } \\
\text { asumido dif. }\end{array}$ & 28,457 &, 000 & $-4,512$ & 15,078 &, 000 & $\begin{array}{l}-1,615 \\
-1,615\end{array}$ & $\begin{array}{l}, 358 \\
, 368\end{array}$ \\
\hline
\end{tabular}

Al analizar esta tabla, en primer lugar, se procede a evaluar el supuesto de varianzas iguales entre los grupos que se están comparando.

En el pretest no hay diferencia significativa entre los grupos p.05. Entonces, las varianzas de ambas muestras son iguales.

En relación con el post-test inmediato p<.05. Por lo tanto, se demuestran los resultados a favor de la estrategia metalingüística, al igual que en el post-test diferido p<.05.

De acuerdo con las hipótesis planteadas en este estudio, podemos señalar que se comprueba la hipótesis 1 y se rechaza la hipótesis nula, pues sí existe diferencia entre grupos que recibieron feedback (GE1 y GE2). Es por ello que el feedback metalingüístico tiene resultados con significancia a corto y largo plazo sobre el grupo control, que no recibió feedback, y el grupo experimental 1, que fue corregido a través de feedback directo. 
Por lo tanto, para responder a nuestras preguntas de investigación, se puede sostener que la estrategia más efectiva para disminuir errores en el uso de MDO en la escritura de crónicas periodísticas es el feedback correctivo escrito de tipo metalingüístico y focalizado, mientras que la estrategia directa utilizada en el grupo experimental 1 no tuvo diferencias estadísticamente significativas, al igual que el grupo control.

\section{Discusión y conclusiones}

Los hallazgos muestran la factibilidad de la operacionalización y adecuación de un modelo utilizado con asiduidad en una segunda lengua para determinar la efectividad del feedback metalingüístico en oposición al feedback directo (Ferreira, 2015, 2017), pero que en este ámbito apoya la escritura de un género académico en español como lengua materna en un contexto mediatizado por la tecnología.

En relación a la pregunta de este estudio que establece los efectos del FCE podemos señalar que los resultados son alentadores, pues el desarrollo de tareas de escritura corregidas mediante estrategias de feedback correctivo escrito metalingüístico conduce a mejorar la escritura a través de la obtención de una correcta precisión en el uso de marcadores discursivos con valor organizativo y a que esto se mantenga en el tiempo, lo que se condice con los estudios a nivel nacional de Ortiz y Ferreira (2014), quienes al comparar estrategias metalingüísticas directas e indirectas concluyeron la supremacía de estas últimas. Además, el estudio realizado por Muñoz y Ferreira (2017) estableció que estas estrategias también eran pertinentes para trabajarlas en enseñanza básica y que el feedback metalingüístico era más efectivo en este escenario.

A raíz de lo anterior, las estrategias correctivas, de acuerdo a Ferris (1999), son pertinentes para mejorar la producción escrita, en oposición a lo planteado por Truscott (1996), quien señalaba que el uso de estas estrategias podría ser perjudicial. En este caso, el feedback correctivo escrito metalingüístico (FCM) mediante descripciones gramaticales fue el que alcanzó mayor éxito, pues este grupo inició el proceso de escritura con 41 errores, pero en el post-test disminuyó a 9 y en el post-test diferido el número de errores fue solo 3, lo que equivale a una mejora con significancia en la disminución de errores en el uso de MDO, lo que se corrobora en las pruebas estadísticas aplicadas en relación con el post-test inmediato p<.05, por lo que se demuestra que los resultados favorecen el uso de la estrategia metalingüística, al igual que en el post-test diferido $p<.05$.

En cuanto a las preguntas de investigación, pudimos constatar que la estrategia más efectiva para la disminución de errores en el uso de MDO corresponde a las claves metalingüísticas, lo que corrobora nuestra segunda hipótesis, que señala que hay diferencias entre grupos y que el grupo que fue corregido mediante FCM presentaría una disminución de errores, lo que se corroboró con significancia estadística p<.05. Sin embargo, en relación a la 
segunda pregunta, no pudimos establecer una mejora significativa en los estudiantes que recibieron feedback correctivo directo, en relación al grupo que no recibió feedback (GC).

Los resultados de esta investigación, además de dar cuenta de la efectividad de la corrección de errores mediante estrategias de FCE, nos permiten conocer y aplicar diversas estrategias correctivas en distintos géneros académicos y profesionales. Cabe señalar que los resultados dan cuenta de la efectividad de la clave metalingüística en oposición a la corrección directa. Esto resulta interesante, debido a que los docentes de español como Lı que trabajan en el aula, al no contar con una taxonomía de errores y estrategias de corrección, solo con los lineamientos entregados por Cassany (2014), corrigen el error de manera explícita y proveen de la respuesta correcta. Lo que nos lleva a esbozar dos hipótesis: primero en relación con la efectividad de la corrección y luego a preguntarnos si los docentes efectivamente saben cómo corregir un texto. En ese sentido, el feedback correctivo escrito podría entregar algunas luces del proceso en el ámbito de español como LI.

A partir de las estrategias utilizadas en este estudio se logró verificar que proporcionar estrategias de feedback correctivo escrito conduce a mejorar el uso de marcadores discursivos con valor organizativo en crónicas periodísticas, pero que la estrategia implícita es la más adecuada para este género discursivo y para esta forma lingüística, lo que se evidenció en el incremento a favor de la estrategia metalingüística en el post-test inmediato y en el post-test diferido.

Para finalizar, se puede sostener que la implementación de tareas en la plataforma Moodle facilitó el trabajo de corrección para el docente y para la recepción del feedback por parte de los estudiantes. En ese sentido, el diseño de las actividades de clases basadas en el enfoque por tareas propuesto por Estaire (2007) permite utilizar un modelo didáctico con un enfoque comunicativo para enseñar lengua en un contexto académico. Desde esa perspectiva, la tarea corresponde a un plan de trabajo que requiere que el estudiante fije su atención principalmente en el significado, haciendo uso de los recursos lingüísticos disponibles (Estaire, 2011), por lo que se une a la necesidad de producir textos en un contexto específico y adecuado para el alumno, entendiendo el entorno natural en el que se desarrolla un estudiante de periodismo, y a la vez propiciar el aprendizaje de marcadores del discurso organizativos para favorecer la redacción de sus textos.

\subsection{Limitaciones y proyecciones del estudio}

Las limitaciones que presenta este estudio dan cuenta de que el feedback correctivo escrito es un tema controversial que puede estudiarse desde diversos ámbitos, ya sea la configuración del género o la variación de la o las formas gramaticales de estudio. Sin embargo, trabajar solo con la noción de feedback en el ámbito de la lengua es un tanto reduccionista, pues resultaría relevante y oportuno proyectar el feedback a la superestructura de la crónica 
para organizar el género discursivo y a nivel macroestructural, con el propósito de entregar feedback en el contenido del escrito, debido a que es una función básica y necesaria para los procesos de textualización y revisión de la escritura.

Otra limitación guarda relación con la elaboración de los test, pues si bien es cierto que estos corresponden al mismo género periodístico, al variar la temática, esto podría influir en la elicitación del uso de marcadores discursivos con valor organizativo.

Es importante señalar que la revisión de la corrección por parte de los estudiantes es fundamental para el éxito en la aplicación de estrategias de FCE; en ese sentido, para efectos de esta investigación se utilizó un foro en el que los alumnos registraban de manera obligatoria sus comentarios respecto al feedback, esto porque la redacción de textos nuevos impide la revisión del texto anterior durante la misma sesión de trabajo. Este punto es interesante de discutir, porque aún no hay resultados contundentes en relación con la escritura y reescritura por parte de los alumnos, y la efectividad del feedback en estas situaciones. De acuerdo con este punto, esta investigación abordó la escritura de textos nuevos; no obstante, trabajos en el área (Ortiz y Ferreira, 2014; Lillo y Sáez, 2017) sostienen que es importante que los estudiantes reescriban el mismo texto para modelar la pertinencia del FCE.

Asimismo, es oportuno indagar en la comprensión de las estrategias metalingüísticas por parte de los estudiantes. Es por ello que sería interesante ahondar en protocolos en voz alta en la reescritura de sus textos una vez que el docente les ha proporcionado feedback para conocer su efectividad, así como también en entrevistas en profundidad con recuerdos evocados para proveer más información cualitativa respecto a la comprensión y eficacia del feedback metalingüístico por parte de los escritores de los textos.

\section{Bibliografía citada}

BITCHENER, John, 2008: "Evidence in support of corrective feedback", Journal of Second Language Writing 17, 102-118.

BITCHENER, John, 2017a: "Creating an effective argument in different academic genres. A scaffolded approach" en John Bitchener, Neomy Storch y Rosemary Wett (eds.): Teaching writing for academic purposes to multilingual students: Instructional approaches, New York: Routledge.

BITCHENER, John, 2017b: "Direct versus indirect feedback" en TESOL Encyclopaedia, New York: Wiley Publishers.

BLEYHL, Werner, 2009: "The hidden paradox of foreign language instruction or: which are the real foreign language learning processes?" en Thorsten PISkE y Martha Young-Scholten (eds.): Input Matters in SLA, Bristol: MPG Books Ltd., 137-158. 
Carlino, Paula, 2013: "Alfabetización académica diez años después", Revista Mexicana de Investigación Educativa 18 (57), 355-381.

Carlino, Paula, 2004: "Escribir y leer en la universidad: responsabilidad compartida entre alumnos, docentes e instituciones" en Paula CARLIno (coord.): Leer y escribir en la universidad, Colección Textos en Contexto 6, Buenos Aires: Asociación Internacional de Lectura / Lectura y Vida, 5-21.

Cassany, Daniel, 2014: Reparar la escritura. Didáctica de la corrección de errores, México: Graó.

COYLE, Yvette, y Julio RoCA DE LARIOS, 2014: "Exploring the role played by error correction and models on children's reported noticing and output production in a 12 writing task", Studies in Second Language Acquisition 36 (3), 451-485.

Chaudron, Craig, 1977: “A descriptive model of discourse in the corrective treatment of learners' errors”, Language Learning 27 (1), 29-46.

Doughty, Catherine, y Jessica Williams, 1998: "Pedagogical choices in focus on form" en Catherine Doughty y Jessica Williams (eds.): Focus on form in classroom second language acquisition, Cambridge: Cambridge University Press, 197-261.

ElLIS, Rod, 2009: "A Typology of written corrective feedback types”, ELT Journal 63 (2), 97-107.

Eluıs, Rod, 2005: “Principles of instructed language learning”, System 33 (2), 209-224.

ERRÁzuriz, María Constanza, 2012: "Análisis del uso de los marcadores discursivos en argumentaciones escritas por estudiantes universitarios", Revista Perfiles Educativos XXXIV (136), 98-117.

EstaIRE, Sheila, 2007: “La enseñanza de lenguas mediante tareas: principios y planificación de unidades didácticas" [http://www.nebrija.es/espanolparainmigrantes/flash/ensenar/PDF/ articulo-tareas.pdf, fecha de consulta: 21 de noviembre de 2016].

EstaIRE, Sheila, 2011: "Principios básicos y aplicación del aprendizaje mediante tareas", MarcoELE: Revista de Didáctica Español como Lengua Extranjera 12 [http://marcoele.com/descargas/12/estaire-tareas.pdf].

Ferreira, Anita, 2017: "El efecto del Feedback Correctivo para mejorar la destreza escrita en ELE", Colombian Applied Linguistics Journal 19 (1), enero-junio, 37-50.

Ferreira, Anita, 2015: "El efecto de estrategias de feedback correctivo escrito en la precisión lingüística de aprendices de ELE” en Actas del XXVI Congreso Internacional de ASELE (Granada, España, 16-19 de septiembre de 2015), 345-355. 
FERRIS, Dana, 2010: "Second language writing research and written corrective feedback", Studies in Second Language Acquisition 32, 181-201.

FERRIS, Dana, 1999: "The case for grammar correction in L2 writing classes: A response to Truscott (1996)", Journal of Second Language Writing 8, 1-10.

HyLAND, Ken, y Fiona HyLAND, 2006: “Feedback on second language students' writing”, Language Teaching 39, 83-101.

LI, Shaofeng, Yan Zhu y Rod ElLIS, 2016: "The effects of the timing of corrective feedback on the acquisition of a new linguistic structure”, Modern Language Journal 100, 276-295.

LILLo, Jorge, y Katia Sáez, 2017: "La efectividad del feedback correctivo escrito en la adquisición de segundas lenguas", Revista Signos 50 (94), 217-240.

LILlo, Jorge, y Anita FerReIRA, 2014: “Un modelo teórico-metodológico para investigación empírica en feedback correctivo escrito en una segunda lengua”, Onomázein 30, 90-110.

LILLo, Jorge, 2014: Efecto del feedback correctivo escrito directo focalizado, en el proceso de producción de textos escritos en inglés como L2. Tesis de doctorado, Universidad de Concepción, Chile.

Long, Michael, 2007: "Recasts in SLA: The story so far" en Michael H. Long: Problems in SLA, Mahwah, NJ: Lawrence Erlbaum Associates, 75-116.

LonG, Michael, 1996: "The role of the linguistic environment in second language acquisition" en William C. Rıtchie y Tej K. Bhatı (eds.): Handbook of second language acquisition, Nueva York: Academic Press, 413-468.

LonG, Michael, 1991: "Focus on form: A designfeature in language teaching methodology" en Kees de Bot, Ralph Ginsberg y Claire Kramsch (eds.): Foreign language research in cross-cultural perspectives, Amsterdam/Philadelphia: John Benjamins Publishing Company, 39-52.

Loewen, Shawn, 2012: "The Role of Feedback” en Sussan Gass y Alison Mackey (eds.): The Routledge Handbook of Second Language Acquisition, Abingdon: Routledge, 24-40.

Martín Zorraquino, María Antonia, y José Portolés, 1999: “Los marcadores del discurso” en Ignacio Bosque y Violeta Demonte (eds.): Gramática descriptiva de la lengua española, tomo III, Madrid: Espasa Calpe, 4051-4203.

Muñoz, Belén, y Anita FerreIRA, 2017: "El feedback correctivo escrito indirecto en el aprendizaje de la forma comparativa de adjetivos en inglés”, Logos: Revista de Lingüística, Filo- 
sofía y Literatura 27 (1), 73-89 [disponible en http://revistas.userena.cl/index.php/logos/ article/view/828].

Murillo, Silvia, 2000: "Los marcadores del discurso y su semántica” en Óscar Loureda y Esperanza Acin (coords.): Los estudios sobre marcadores del discurso en español, hoy, Madrid: Arco Libros, 241-280.

Ortiz, Mabel, y Anita FerReIRA, 2014: "Proposing a Wiki-Based Technique for Collaborative Essay Writing”, Profile: Issues in Teachers' Professional Development 16 (2), 185-198.

Pons, Salvador, 1998: Conexión y conectores. Estudio de su relación en el registro informal de la lengua, Cuadernos de Filología, Anejo XXVII, Universidad de Valencia.

Portolés, José, 2014: Marcadores del discurso, Barcelona: Ariel.

Pottier, Bernard, 1993: Semántica general, Madrid: Gredos.

RaZAGIFARD, Parisa, y Vida RazzAghifard, 2011: "Corrective feedback in a computer-mediated communicative context and the development of second language grammar", Teaching English with Technology 11 (2), 1-17.

Salaberri, M. a Sagrario, y Ana Mónica Altamirano, 2001: "Instruction in learning strategies" en M. ${ }^{a}$ Elena Garcí (coord.): Present and future trends in TEFL, Almería: Servicio de Publicaciones de la Universidad de Almería.

SHEEN, Younghee, 2011: Corrective Feedback Individual Differences and Second Language Learning, New York: Springster.

Shintani, Natsuko, Rod Ellis y Wataru SuzukI, 2014: "Effects of written feedback and revision on learner's accuracy in using two English grammatical structures", Language Learning 64 (1), 103-131.

Silva Corvalán, Carmen, 2001: Sociolingüística y pragmática del español, Washington, D.C.: Georgetown University Press.

TRuscotT, John, 1996: "The case against grammar correction in 12 writing classes", Language Learning 46 (2), 327-369.

Truscott, John, y Angela Hsu, 2008: "Error correction, revision, and learning”, Journal of Second Language Writing 17 (4), 292-305.

Van DıJk, Teun, 1980: Estructuras y funciones del discurso. Una introducción a los estudios del discurso, México: Siglo XXI Editores. 